Man and Nature

L'homme et la nature

\title{
Voltaire et La Mort de César
}

\section{Georges-L. Bérubé}

Volume 1, 1982

URI : https://id.erudit.org/iderudit/1011787ar

DOI : https://doi.org/10.7202/1011787ar

Aller au sommaire du numéro

Éditeur(s)

Canadian Society for Eighteenth-Century Studies / Société canadienne d'étude du dix-huitième siècle

ISSN

0824-3298 (imprimé)

1927-8810 (numérique)

Découvrir la revue

Citer cet article

Bérubé, G.-L. (1982). Voltaire et La Mort de César. Man and Nature / L'homme et la nature, 1, 15-20. https://doi.org/10.7202/1011787ar

Copyright (c) Canadian Society for Eighteenth-Century Studies / Sociéte canadienne d'étude du dix-huitième siècle, 1982
Ce document est protégé par la loi sur le droit d'auteur. L'utilisation des services d’Érudit (y compris la reproduction) est assujettie à sa politique d'utilisation que vous pouvez consulter en ligne.

https://apropos.erudit.org/fr/usagers/politique-dutilisation/ 


\section{Voltaire et La Mort de César}

Dans l'esprit de Voltaire, comme dans celui d'un certain nombre de ses contemporains, ${ }^{1}$ La Mort de César représentait une hardiesse telle que l'auteur ne s'étonna guère de devoir attendre plus de dix ans avant de voir sa tragédie jouée sur le Théâtre français. ${ }^{2}$ La suppression des intérêts amoureux et des rôles féminins - nous connaissons l'influence prépondérante des comédiennes de l'illustre compagnie dans le choix des pièces, ainsi que le goût du public de l'époque - , l'altération de la forme de la tragédie - La Mort de César n'avait que trois des cinq actes prescrits par la dramaturgie classique, - l'interprétation nouvelle d'un mythe séculaire faisant de Brutus le fils de César plutôt qu'un simple conjuré et, enfin, l'idéologie de la pièce, suspecte parce qu'inspirée trop ouvertement d'un modèle anglais qui était jugé révolutionnaire en France à ce moment-là, voilà autant de nouveautés dont Voltaire crut pouvoir s'enorgueillir. Quelque osées que fussent ces innovations et bien que chacune eût sans doute son importance dans l'esprit de Voltaire, une seule retiendra notre attention aujourd'hui, soit l'élimination de l'intrigue amoureuse et la prétention de l'auteur d'avoir réussi, par là, à "guérir le théâtre français de cette contagion [l'amour]," autrement dit, à purger le théâtre des galanteries qui l'affadissaient. Dans sa correspondance et dans ses écrits, Voltaire revient sans cesse sur l'idée qu'il faut opérer une révolution qui débarrasse le théâtre des fadaises amoureuses et détrôner, par conséquent, le despote dont parle le comte Algarotti dans sa lettre à l'abbé Franchini: "L'amore è signor despotico delle scene francesi ${ }^{3}$." A notre humble avis, si la pièce relate le renversement d'un despote appelé César, elle ne réussit pas pour autant à détruire l'amore despotico, ni même à purger le théâtre français.

Avant d'aborder le sujet proprement dit, rappelons brièvement l'action de La Mort de César. Après avoir subjugué Rome, César est sur le point de se proclamer roi, lorsqu'il avoue à Antoine que Brutus, ce républicain féroce, ennemi des tyrans, est son propre fils qui s'ignore. S'opposant aux conseils d'Antoine qui préconisait l'utilisation de la force pour imposer son règne, César entreprend de se faire aimer de Brutus et du Sénat. A l'acte II, Brutus dénonce avec violence les projets politiques de César et, devant ses amis alarmés, il s'engage à tuer quiconque voudrait détruire la République et la liberté romaine. Survient César, qui produit une lettre prouvant incontestablement à Brutus qu'il est le fils de son ennemi implacable. Abasourdi par cette révélation, Brutus s'acharne tout de même à ne pas reconnaître son père dans le tyran de Rome. A l'acte III, Brutus apprend aux 
conjurés le secret qui le ronge; Cassius, porte-parole des rebelles, déclare que le sang n'est rien, que seul l'amour de Rome doit remplir le coeur d'un citoyen. Brutus, dans un ultime effort pour dissuader son père, lui avoue qu'il l'aime. Mais César brave les menaces; il meurt assassiné par les conjurés. La pièce se termine par la harangue d'Antoine aux Romains les engageant à punir les coupables.

Outre le fait que, visiblement, la distribution ne comporte pas de rôle pour les femmes et, qu'en apparence du moins, l'auteur se préoccupe uniquement du débat politique mettant aux prises un républicain et un monarchiste, nous ne croyons pas que cela suffise à prouver l'expurgation du théâtre et la défaite de l'amour galant telles que claironnées par Voltaire. Bien au contraire, les critiques relevèrent avec justesse que l'amour, loin d'être banni, s'était métamorphosé. A cet effet, dans l'introduction à son édition de La Mort de César, André Rousseau propose l'idée de transmutation:

La parenté de César et Brutus, très vague soupçon chez Plutarque et Shakespeare. . . s'étale ici avec une complaisance significative. Non seulement se trouve réintroduit le sentiment que l'absence des femmes avait éliminé, mais Voltaire donne un nouvel exemple de sa conception "sentimentale" de la tragédie et du tragique. . . ."

Quelle idée attrayante que d'établir la continuité entre les premières tragédies (p. ex. OEdipe, Artémire) et La Mort de César en posant l'hypothèse que l'amour "galante" des premières se transforme en amour paternel dans la deuxième. Idée intéressante, soit, mais qui se heurte à des objections dès que l'on tente de la vérifier, car ce n'est pas tout de rapprocher La Mort de César aux premières tragédies, encore faut-il s'assurer que nous comparons des choses de même nature, susceptibles d'être comparées: la relation Philoctète - Jocaste (OEdipe), c'est-à-dire l'amour entre un homme et une femme, disparaît-elle dans les oeuvres subséquentes, transformée en amour paternel (p. ex. Brutus - César)? Ou se maintient-elle sous une forme moins évidente, moins visible? La nécessité d'une théorie de la transmutation dépend de la réponse apportée à cette question. Nous aurons l'occasion d'y revenir.

En fait, cette théorie de la transmutation fut reprise et développée par G. Defaux dans une analyse des idées politiques de Voltaire dans La Mort de César. ${ }^{5}$ Le critique l'explique ainsi:

l'amour, qu'il [Voltaire] avait chassé avec éclat par la porte, revient en catimini par la fenêtre. Chez Voltaire, Brutus n'est pas seulement le champion de la Liberté romaine, il est aussi le fils de César. L'auteur voulait par ce moyen compenser l'absence d'amour dans sa tragédie; il ne fait en définitive que réintroduire ce sentiment sous une autre forme. ${ }^{6}$

Pour Defaux, ce palliatif qu'est le retour subreptice de l'amour, s'il pouvait compenser "la sécheresse inhérente à tout débat d'idées," n'entraînait pas moins la tragédie sur la pente du mélodrame; le critique conclut: "le conflit Liberté - Tyrannie. . .prend chez Voltaire l'étroitesse d'une moralité pleurarde, il s'efface devant le drame familial."

Un autre chercheur, Robert Cottrell, signale lui aussi la transmutation de 
l'amour dans La Mort de César, tout en attirant notre attention sur un aspect négligé jusqu'à présent: le langage.

Others have noted . . . that the tender but anguished relationship between César and Brutus replaces the more traditional love intrigue in contemporary tragedies. Indeed the most striking features of La Mort de César is the way in which Voltaire introduces situations and language appropriate to a love story into the economy of his tragedy. ... By eliminating women from La Mort de César he did not rid his tragedy of the contagion of love. The manner in which love, shooed out one door, slips back in through another deserves, I believe, a closer examination. ${ }^{8}$

Après y avoir regardé de plus près, Cottrell est d'avis que l'amour est seul responsable du renversement de César: "The conspirators administer the coup de grâce, but it is love that topples César from his seat of power."' En effet, selon Cottrell, César est aussi amoureux de Brutus que Phèdre pouvait l'être d'Hippolyte. Pour appuyer ses dires, le critique fait appel au langage employé par César pour parler de son fils: "Although Voltaire is here [dans un extrait] describing paternal love, he has couched it in the traditional language of erotic affection ...."10 Ainsi, quelles que soient leurs divergences d'opinions sur d'autres sujets, tous ces critiques s'entendent sur un point précis: l'amour paternel dans La Mort de César est une transmutation de l'amour "galant".

Or, cette opinion semble reposer d'abord et avant tout sur le postulat que nous avons affaire ici à une pièce expérimentale, originale, unique et que, pour cette raison, elle ne se donne pas à lire comme toutes les autres pièces voltairiennes de la même époque. Exception faite de Ronald Ridgway, ${ }^{11}$ les autres critiques comparent La Mort de César aux premières tragédies, mais ils oublient de la replacer dans son contexte historique et chronologique, de telle sorte que leurs interprétations nous donnent l'impression qu'ils crurent Voltaire sur parole lorsqu'il se vante d'avoir expulsé l'amour galant de cette tragédie. Voilà qui explique pourquoi ils durent créer le concept d'une transmutation de l'amour. Que l'amour soit présent dans cette oeuvre, cela ne fait aucun doute, mais la forme que prend cet amour est problématique.

Il est essentiel, quant à nous, de partir du principe que La Mort de César n'est pas une pièce expérimentale, qu'elle contient vraisemblablement tous les éléments qui caractérisent les autres pièces de Voltaire écrites à cette époque, notamment Brutus (1730) et Eriphyle (1731). Par conséquent, notre première démarche qui consiste à superposer ces oeuvres révèle que, dans les deux dernières, un fils tombe en amour avec une femme aimable et séduisante contre le volonté expresse de son père: Titus, dans la pièce Brutus, s'éprend de la princesse Tullie, ce qui le mène à trahir Rome et son père; Alcméon, dans la pièce Eriphyle, s'éprend de la reine, ce qui le mène à transgresser l'interdiction du grand-prêtre et à trahir les dieux et son père. Chaque fois, le fils choisit d'aimer plutôt que de se plier à la volonté paternelle, il opte pour l'amour plutôt que le devoir et la fidélité. Dans La Mort de César, le père veut également récupérer son fils Brutus, le soustraire à l'influence, non d'une femme, mais des conspirateurs; le fils choisit une fois de plus de trahir son père. Malgré cette trahison, peut-on dire que Brutus sacrifie son père à 
l'amour d'une femme? En apparence, rien de tel puisqu'aucune femme n'entreprend de séduire Brutus. Néanmoins, notre démarche met en évidence des rapports récurrents d'une pièce à l'autre, rapports qu'il faut tâcher d'élucider davantage.

Il ressort, entre autres choses, que l'amour d'un père pour son fils, loin d'être une transmutation nouvelle et originale dans La Mort de César comme les critiques cités plus haut ont bien voulu nous le faire croire - cet amour, disions-nous, existe comme rapport récurrent dans plusieurs pièces de cette période et, ce qui est plus révélateur, il s'oppose toujours à l'amour du fils pour une femme, ennemie du père. Outre les tragédies de Brutus et d'Eriphyle, nous pourrions citer les comédies des Originaux ou Monsieur du Cap-Vert (1732), de L'Echange ou Quand est-ce qu'on me marie? (1734) et même un livret l'opéra, Samson (1732). Ainsi, dans La Mort de César, au lieu de fabriquer une quelconque théorie de la transmutation et de faire entrer l'amour "en catimini par la fenêtre", ne serait-il pas plus simple - et surtout plus logique - d'y chercher, en dépit des difficultés, le même conflit entre l'amour hétérosexuel et l'amour paternel que nous avons relevé dans toutes les autres pièces de Voltaire écrites à ce moment-là?

Poursuivons cette idée en nous demandant quelle sorte de femme pourrait bien se cacher dans cette oeuvre, en apparence dépourvue de personnage féminin? D'après les résultats de notre superposition de pièces, les personnages féminins de Voltaire se présentent généralement comme des êtres faibles, prisonniers d'un pouvoir étranger, victimes de manigances politiques, enfin, ils constituent des appâts destinés à leurrer un héros sur son devoir politique. ${ }^{12} \mathrm{Y}$ a-t-il un tel personnage dans La Mort de César? Si la réponse est toujours négative, nous devons alors escompter la manifestation de ces idées sous une forme autre que le personnage.

En effet, depuis E. Souriau et A.-J. Greimas, nous savons que toutes les "fonctions" - dramaturgiques ou actantielles - n'ont pas à être présentes au microcosme théâtral dans un personnage distinct, que, parfois, un objet (p. ex. une couronne) ou une idée peuvent incarner le bien souhaité, etc. D'autre part, la poésie utilise constamment le procédé qui consiste à personnifier des choses inanimées. Alors, pourquoi ne pas chercher l'élément féminin qui contrebalance l'influence du père au niveau textuel, comme s'il s'agissait en fait d'un effet de style? La réponse s'impose alors à notre esprit: grâce à la personnification, l'élément féminin séducteur de Brutus s'appelle Rome. ${ }^{13}$

En réalité, Brutus et les conspirateurs sont les victimes d'un divorce politique puisqu'ils ont à choisir entre César - qui se veut leur père - et Rome qu'ils considèrent déjà comme leur mère. Cassius en dit autant à César: "Avant que d'être à toi nous sommes ses enfants [à Rome]" (I,E). Cassius, lorsqu'il veut convaincre Brutus de persévérer dans le projet d'assassiner César, demande: “Toi, son fils! Rome enfin n'est-elle plus ta mère?" (III,2) Voilà donc le personnage invisible chargé de séduire le fils de César, chargé d'agir comme pôle d'attraction hostile au père: Rome est la femme fatale pour qui Brutus va tout sacrifier.

Comme Tullie et Eriphyle, Rome est un personnage royal, détenu contre son gré: 
Dieux! maîtresse de l'Inde, esclave aux bords du Tibre! Qu'importe que son nom commande à l'univers Et qu'on l'appelle reine, alors qu'elle est aux fers? $(I, 3)$

Comme Tullie et Eriphyle, Rome occupe une position politique diamétralement opposée à celle représentée par l'homme fort au pouvoir: Rome est républicaine, alors que César prétend imposer un régime monarchique; comme Tullie et Eriphyle, Rome est un enjeu politique considérable que chacun s'arrache; comme pour Tullie et Eriphyle, la seule issue pour Rome réside dans la séduction du fils de l'homme fort: Rome doit provoquer Brutus et le tourner contre son père: "Entraîné par César, et retenu par Rome" (III,2), avoue le fils affligé. Rome possède donc toutes les caractéristiques du personnage féminin des autres pièces voltairiennes de l'époque: elle est faible, détenue, appât et rivale.

Par contre, parler de la séduction d'un personnage réel par une abstraction personnifiée dans le texte ne va pas sans créer certains problèmes. Voltaire s'en rendit compte et, pour se tirer d'embarras, dut recourir à un subterfuge peu satisfaisant. Phénomène remarquable, la scène de séduction prend la forme d'un monologue: l'acte II, scène 2. Désemparé devant l'initiative de son père qui veut détruire Rome (tout comme Brutus voulait chasser Tullie et les dieux voulaient détruire Eriphyle), Brutus se lamente: "Et je cherche ici Rome, et ne la trouve plus." Au pied de la statue de Pompée, représentant des vertus romaines, Brutus trouve un billet anonyme: "Tu dors, Brutus, et Rome est dans les fers!" Sans hésiter, Brutus répond à la personne responsable: "Rome, mes yeux sur toi seront toujours ouverts;/Ne me reproche point des chaînes que j'abhorre" (c'est nous qui soulignons). Et comme si ce premier billet ne devait pas suffire, Brutus en aperçoit un second: "Non, tu n'es pas Brutus!' Voilà donc que Rome dialogue avec son amant par billets interposés! A cette accusation de sa maîtresse, Brutus ne résiste plus; il se déclare prêt à venger Rome. Voici de quelle façon il s'y prend:

On demande un vengeur, on a sur moi les yeux;

On excite cette âme, et cette main trop lente;

On demande du sang. . . Rome sera contente.

Incapable de préciser quelle femme exige de lui cet effort suprême, Brutus emploie le pronom impersonnel "on" et termine en assurant que sa maîtresse sera satisfaite. Ainsi, sans l'intervention d'aucune femme, Brutus se trouve séduit par des billets qu'il attribue à l'être aimé. Cassius le lui dira un peu plus tard: "C'est Rome qui t'inspire en des desseins si grands" (II,4).

Cette entité féminine anthropomorphe nous permet de tirer quelques conclusions au sujet de La Mort de César, dont une première, très importante: la présence de Rome consacre l'échec de Voltaire dans sa campagne d'expurgation du théâtre. Malgré ses déclarations et toutes ses intentions révolutionnaires, l'auteur réintroduit, dans une notion abstraite et personnifiée, les éléments féminins qu'il jugeait indésirables, ce qui a pour effet de rendre cette pièce semblable aux autres. Pas de statut expérimental, par conséquent, et nul besoin d'inventer une théorie de la transmutation puisqu'en fait l'oeuvre reprend le grand thème commun à toutes les pièces de 
l'époque, soit le conflit entre l'amour hétérosexuel et l'amour paternel, entre l'amour et le devoir. Placé dans une situation pénible où il doit choisir entre son père et sa mère, le fils opte pour la femme et l'amour, provoquant ainsi la catastrophe finale. L'analogie avec Eriphyle est ici des plus saisissantes: le spectre du père revient sur terre pour empêcher l'union incestueuse du fils et de la mère. La situation est comparable dans La Mort de César où le père tente par tous les moyens de rompre l'attachement malsain de Brutus pour Rome, le tout sans succès: "Rome demande un maître:/Un jour à tes dépens tu l'apprendras peut-être" (III,4). Au dénouement, l'analogie se poursuit: par erreur, Alcméon tue sa mère sur le tombeau de son père et perd l'usage de ses sens; Brutus, quant à lui, permet l'assassinat de son père et, par erreur, plonge Rome dans la guerre civile, perdant alors l'usage de ses sens. Après leur crime, Alcméon, Brutus et même Titus ne sont plus que des loques humaines, victimes trop désireuses du supplice qu'ils ont mérité.

Nous pourrions conclure de la façon suivante: l'amour que Voltaire avait chassé revient, non pas sous forme d'un amour paternel - celui-ci étant déjà présent dans les autres pièces de l'époque -, mais bien dans l'image anthropomorphe de Rome, cette reine "esclave aux bords du Tibre".

Georges-L. Bérubé Université de Victoria

\section{Notes}

' Parmi ceux-là, citons le conte Algarotti, LePelletier, Cideville, Desfontaines, etc. Cf. par exemple Best. D426 et D909.

${ }^{2}$ Commencée en Angleterre, La Mort de César fut complétée en juin 1731 (Best. D417); elle eut quelques représentations privées avant d'être créée à la Comédie française le 29.8.1743.

3 Algarotti, Lettera del signor conte Algarotti, publiée avec la tragédie de La Mort de César.

${ }^{4}$ André Rousseau, Voltaire: La Mort de César (Paris: S.E.D.E.S., 1964,), p. 19.

'G. Defaux, "L'Idéal politique de Voltaire dans La Mort de César," Revue de l'Université d'Ottawa, 40, No. 3 (1970), 418-440.

6 Defaux, p. 428.

Defaux, p. 428

${ }^{8}$ Robert Cottrell, "Ulcerated Hearts: Love in Voltaire's La Mort de César," dans Literature and History in the Age of Ideas, Ch. Williams éd. (Columbus; Ohio State Univ. Press, 1975), p. 171 .

${ }^{9}$ Cottrell, p. 175.

${ }^{10}$ Cottrell, p. 174.

1 Cf. Ronald Ridgway, La Propagande philosophique dans les tragédies de Voltaire, SVEC 15 (1961), 84 .

12 Tullie, dans Brutus, est prisonnière de Rome, tout en étant un enjeu politique dont se servira Arons pour séduire Titus. Eriphyle est prisonnière dans son propre palais; elle finit par obtenir l'aide d'Alcméon malgré l'interdiction formelle des dieux.

${ }_{13}$ Ainsi, l'idée d'une transmutation dans laquelle l'amour changerait d'objet - l'amour hétérosexuel qui devient amour paternel - est inutile; il s'agit toujours, dans cette pièce, du même amour hétérosexuel, mais certains procédés d'écriture en ont modifié la manifestation. 